\title{
Is the human species special? It is... in its quest for specialness
}

\author{
Zina Skandrani
}

Published online: 18 June 2013

(C) AESS 2013

In the global environmental and biodiversity crisis, nature conservation efforts often focus on the treatment of symptoms rather than on the source of the collapse. How humans shapeor, as the case may be, degrade - their environment certainly depends on their industrial, agricultural and resource exploitation systems. But even deeper, all these practices of environmental transformation are ruled by humans' cultural systems of representations. For instance, it has been recognized long ago that the way people think of themselves as apart from nature has negative effects on the way they deal with and treat their environment. These dualistic human/nature conceptions, however, are in turn grounded on other representations, and central among them is the permanent assertion of human's uniqueness among the living.

Arguments surrounding the specialness of the human species are subject to a constant renewal through the continuous supplying of new and less new evidence. Human culture/civilization, human's mastery of or achievement on earth, human intelligence/rationality, the capacity of language, etc. are among the most prominent and frequent claims of human specialness. It is worth noting, however, that there is not so much a debate about the question as a contest for finding the ultimate and conclusive argument. I will argue that despite its widely accepted evidence, this is not only a hazardous question to be occupied with, but also nonsense.

Surprisingly, the arguments about human specialness ignore their inherent vicious circle (petitio principii). Indeed when comparing the species, human specialness supporters extrapolate some attribute unique to humans or another-i.e., an attribute that only humans share or have more than other species (as the examples mentioned above, of culture,

\section{Z. Skandrani $(\bowtie)$}

Laboratoire de Conservation des Espèces, Restauration et Suivi des

Populations, Museum National d'Histoire Naturelle, 55, rue

Buffon, 75005 Paris, France

e-mail: skandrani@mnhn.fr rationality, language etc.), and declare them as accounting for human specialness. In so doing, they elevate specifically human characteristics as criteria to measure specialness per se. However, these criteria, while important for the human species itself, bear no independent relevance outside the human reference system. The fallacious reasoning consists, then, in that what has to be proven (human specialness) is implicitly assumed in the premises (human characteristics as specialness criteria). In a more illustrative way, this homocentric perspective would be equal to and no more legitimate than the specialness assessment of the Cheetah species (Acinonyx jubatus) from Cheetahs' point of view, based on their unequaled celerity, or of vegetal species from a plant's point of view, based on its capacity to do the photosynthesis. Of course, these characteristics are never put forward as measures of specialness for humans, because they bear no special relevance to them. These examples demonstrate that there are characteristics that are unique to a species without necessarily accounting for a species' specialness, joining herewith what Darwin already termed in 1871 differences "of degree and not of kind". 1

In order to avoid arbitrariness, definitions of specialness, to be legitimate, would have to rely on a well-defined criterion that is common and relevant to all species in their own reference systems. One such criterion with all species encompassing relevance could be, for instance, the principle of survival. From this perspective, however, humans wouldn't be more special than any other species in the present time. Similarly for every other criterion that could be found, precisely because of its relevance for all species, its selection would necessarily imply (according to the principles of evolution) that it is favored among all survived species. The impossibility of finding a valid criterion that enables the affirmation of a species' uniqueness does not

\footnotetext{
${ }^{1}$ Darwin. C (1871) : The Descent of Man, and Selection in Relation to Sex. London: John Murray.
} 
only deny humans a special place among the living, it also shows that the question of human specialness is nonsense, containing at the outset its own refutation.

The continuous questioning of human specialness reveals, therefore, to be less a genuine epistemic question than an ideological striving. The starting point is not an open question but an attempt to prove an a priori conviction. Humans' claim to specialness is anchored far back in monotheistic religions, placing man in the center of the world or "creation": it has been subsequently legitimated over history by different theologies and dominant systems of belief, which did not tolerate contradiction. Nowadays, there has been no break-off with this, only a slight shift, in the sense that the current dominant belief system, science, has replaced (at least in the western world) the precedent ones in the justification and legitimation of the human specialness ideology. Among the first, inconspicuous emergences, is, to note Linneus' qualification of humans, parallel to their (groundbreaking) assignment to the primate genus, as sapiens. In so doing, he introduced the taxonomic classifications, usually relying on exclusively biological criteria, a qualitative aspect as difference defining humans as a species.

The remaining question is why humans can't accept being a particular species among other equally particular species. A glimpse to other human imprinted divisions shows how profound this quest for difference is. The human affirmation of uniqueness is indeed only a mirror of other common oppositions and comparisons of the self vis-à-vis others, from the macro- to the micro-level: earth/other planets, own culture/others' culture, own country-city/others' countrycity, we/they, me/you, to only name a few. It thus appears that the thesis of human specialness, in the same way as the other mentioned oppositions, results less from a naturally given ordering of the living then from a human attempt to structure human life and ultimately the world. Further, the continuous production of the human/other living distinction continuously builds human identity, an identity constituted by exclusion. The human specialness leitmotiv has in this way hijacked other life-forms to define humans and their own status, revealing less about humans' place among the species than about humans themselves.

It remains, however, that the human specialness ideology promptly becomes an obscurantist barrier to the urgent improvement and change in humans' current actions on the environment, as soon as this advancement means challenging humans' privileged place. And it belongs uniquely to humans to become aware of this, for the sake of all the earth's uniquenesses.

Acknowledgments I would like to thank Dr. Omri Böhm and Dr. Assaf Shwartz for their kind help and comments in revising the manuscript. 